\title{
Segmentation of images by variational methods: a constructive approach ${ }^{1}$
}

\author{
JEAN-MICHEL MOREL and SERGIO SOLIMINI
}

ABSTRACT. We give a constructive proof that given a bounded function on a rectangle $R$, the minimum of the following functional is achieved:

$$
E(u, B)=\|u-g\|_{L^{2}(R)}+l(B),
$$

where $B$ is a finite set of $C^{1}$ curves in $R$ and $u$ is locally constant in $R-B$. In image processing, $g$ can be interpreted as an image $(g(x, y)$ is the grey level at $(x, y))$ and the curves of $B$ as the contours of the image, $u$ representing the mean value inside each contour. Following ideas of Mumford and Shah, our proof suggests a method for transforming an image into a cartoon.

\section{INTRODUCTION AND NOTATION}

One of the central problems in image processing (and more generally in signal or data analysis) is the so called «segmentation problem».

By an (image) we mean (we adopt a continuous deterministic model) a real function $g$ on an open rectangle $R$ in $\mathbb{R}^{2} ; g(x, y)$ is the sgrey-level» at the point $(x, y)$. An «image segmentation» is a pair $(u, B)$ where $B$ is a set of piecewise $C^{1}$ curves, which we call «contours», and $u$ a real function which is regular on connected components of $R / B$.

In a "good» segmentation $(u, B)$, the curves of $B$ should be the boundaries of homogeneous areas in the image and $u$ a sort of mean or, more generally, a regularized version of $g$ in the interior of such areas.

1 Aknowledgement. Work partially supported by US Army under Contract DAJA45-88-C0009.

1980 Mathematics Subject classification (1985 revision): 68U10, 68U05, 65D07, 49 A99.

Editorial de la Universidad Complutense. Madrid, 1988. 
A full account of this problem can be found, e.g., in Rosenfeld and Kak [8]: many algorithmic approaches have been defined about how to define $u$ and $B$, but only very recently the work of S. and D. Geman [4] has suggested that many tasks in image processing should be achieved by minimizing some ad hoc energy functional defined on a set of pictures. This idea has been developped by D. Mumford and J. Shah [7] in a deterministic framework. They propose a global variational method for boundary detection in images, motivated by the failure of present local methods for giving psychologically reasonable contours in some non pathological cases.

According to the simplest version of this model, a segmentation of $g$ is a pair $(u, B)$ where $B$ is a finite set of piecewise $C^{1}$ noncrossing curves from $[0,1]$ into $R$ (with some ends eventually on the boundary of $R$ ), and $u$ is locally constant on $R / S(B)$. $S(B)$ denotes the support of $\mathrm{D}$. Mumford and Shah have proved in [6] the following theorem:

Theorem. Let $g$ be a measurable bounded function in $R$. Then the minimum of the following functional is attained at some $B$ :

$$
E(u, B)=\|u-g\|_{L^{2}(R)}+\text { length }(B)
$$

The curves of the minimum $B$ are in fact $C^{1}$ for an ad hoc parametrization and meet each other only at triple points with $120^{\circ}$ angles. They can also end at the boundary of $R$ with $90^{\circ}$ angles. If $g$ is continuous, each interior point $x$ of $a$ curve $c$ verifies the following Euler-Lagrange equation:

$$
\text { (Curvature of } c \text { at } x)=\left(j u m p \text { of }(u-g)^{2} \text { accross } c \text { at } x\right)
$$

In these notes we shall give an elementary constructive proof of this theorem. Our proof has been obtained independently, as the preceding result was announced, but unpublished. Its scope is also very different. The proof of Mumford and Shah [6] is based on geometric measure theory. Can the model lead to a computational approach? In other words, how can we define and handle minimizing sequences with good computational properties? A first answer to this question has been to give a proof of the preceding result providing useful tools for handling segmentations made of affine curves along a minimizing sequence: we shall consider a minimizing sequence $B_{n}$ of segmentations made of piecewise affine curves and give a priori lower bounds on the areas of the connected components of $R / S\left(B_{n}\right)$. We also deduce upper bounds on the number of curves defining $B_{n}$ and on the number of geometrical crossings of $B_{n}$ and prove that all these estimates pass to the limit. These estimates are not true for any minimizing sequence. One has to work with minimizing sequences made of affine curves and which, from the image processing viewpoint, have some «nonmerging property": The "nonmerging property» is that no boundary between two homogeneous zones of 
the segmentation can be removed without increasing the energy. An affine segmentation being given, this property can be achieved in a constructive way by eventually removing the non useful boundaries. (The only reason for which we choose to consider segmentations made of affine curves is that adding a segment to such segmentations cannot create infinitely many crossings).

Numerical analysis. The preceding result and the lemmas given next suggest a method for computing a minimizing segmentation. The algorithm should works as follows:

- Introduce some reasonable initial segmentation (for instance given by the classical method of zero-crossings of the Laplacian).

- Merging for obtaining the nonmerging property mentioned above.

- Modifying the boundaries in order that the «elasticity" condition about the curvature is verified.

This describes a deterministic gradient procedure. In order to attain a good minimum, one should split again stochastically the regions by adding new edges, and test wether the nonmerging property is still verified. This would correspond to an annealing-like method [4].

Numerical tests are beginning now.

Validity of the method. It will be of course decided by practical experiments. Some researchers in image processing have suggested to us that the original signal to be segmented should be the absolute value of the gradient of $g$, rather than the grey levels $g(x, y)$. Now, whatever may be the form and meaning of the signal to be segmented, it is clear that a global variational approach will involve a functional with one- and two-dimensional terms. Among such functionals, the functional which we shall study is clearly the simplest one!

A related work. After this work was complete, we learned that a researcher of Harvard, Yang Wang [9], has made another constructive proof of the above theorem. The method of Yang Wang is different from ours but has also its computational interest: he defines a discretisation of the minimization problem, with restrictions on the number and length of the segments, and obtains a priori bounds which allow to pass to the limit.

Contents. We shall prove in section 1 that $E$ is lower semicontinuous for a suitable topology.

In section 2 we consider a minimizing seçuence $B_{n}$ of segmentations made of piecewise affine curves and give a priori lower bounds on the areas of the connected components of $R / S\left(B_{n}\right)$.

In section 3 we deduce upper bounds on the number of curves defining $B_{n}$ and on the number of geometrical crossings of $B_{n}$. Section 4 is devoted to the 
proof that all these estimates pass to the limit along a minimizing sequence and concludes the proof of the theorem.

We begin with some notations and definitions which will be useful in the following.

The function $g$. It is a bounded measurable real function on a rectangle $R$. It represents the grey levels of a rectangular image. Without loss of generality we shall assume that $|g(x, y)| \leqslant 1$.

The energy $E(B)$. Note that given $B$, the corresponding minimal $u$ is completely defined by the fact that its value on each connected component of $R / S(B)$ is equal to the mean value of $u$ on this connected component. Thus we shall always assume in the following that to each $B$ is associated this unique $u$. Therefore we shall write $E(B)$ instead of $E(u, B)$.

Length of a curve $c$ from $[0,1]$ into $R$. It is the minimal Lipschitz constant of $c o s$ where $s:[0,1] \rightarrow[0,1]$ is a reparametrization of $c$. By Ascoli-Arzela theorem this minimum, if finite, is achieved. If $c$ is one to one, the length $l(c)$ of $c$ can be equivalently defined as the supremum of the lengths of all piecewise affine curves whose vertices form a finite sequence $c\left(s_{1}\right), \ldots, c\left(s_{k}\right)$ with $s_{1}<s_{2}<\ldots<s_{k}$. In what follows, we shall always deal with Lipschitz curves.

Tips of a curve $c . c(0)$ and $c(1)$.

Interior points of a curve. All other points of the range of $c$.

Noncrossing curves. $c$ is noncrossing if its restriction to $] 0,1[$ is one to one. The curves $c_{i}$ and $c_{j}$ are noncrossing if their interiors do not meet.

Piecewise affine curve. A curve whose support is made of a finite number of segments.

Segmentation $B$. By segmentation we denote a finite family of noncrossing Lipschitz curves, $B=\left(c_{k}\right)_{k}$. If they are piecewise $C^{1}$, we shall call it $C^{1}$.

Segmentation. If they are piecewise affine, we shall call it piecewise affine segmentation.

Geometrical support of $B$. It is denoted by $S(B)$ and is the union of the ranges of the $c_{k}$.

Geometrical crossings of $B$. All the points of $S(B)$ at which $S(B)$ is not locally a one dimensional continuous manifold. Since the curves of $B$ are one to one: $] 0,1[\rightarrow R$, they are simply all the points of the closure of $R$ where a 
curve meets the interior of another, where at least three curves have a common tip, where a curve meets the boundary of $R$.

Geometrical curves of $\mathbf{B}$. All the one dimensional submanifolds of $B$ which have their boundary contained in the set of geometrical crossings of $B$ and which contain no geometrical crossing in their interior.

Length of $B$. Let $B=\left(c_{k}\right)_{k}$ be a finite family of curves ( $B$ is not necessarily a segmentation). By length of $B, l(B)$, we mean the minimun of the sum of the lengthes of the $c_{k}$ :

$$
l(B)=\min \Sigma_{k} l\left(c_{k}\right)
$$

this minimum being taken among all possible representations of $S(B)$ as the union of the ranges of Lipschitz curves $c_{k}$. If $B$ is a segmentation, its length is the sum of lengths of its curves.

Connected components of $\mathbf{R} / \mathbf{S}(\mathbf{B})$. If $B$ is made of piecewise affine curves, their number is finite. We shall denote them by $\left(0_{i}\right)_{i}$ and call them "areas».

Common boundary of two areas $0_{i}$ and $0_{j}$. We denote it by $b\left(0_{i}, 0_{j}\right)$. It is contained in $S(B)$. If $i=j, b\left(0_{j}\right)$ denotes the boundary of $0_{j}$.

Two dimensional measure of $0_{i *}$ Denoted by $\left|0_{i}\right|$.

E-neighbourhood of a subset $\mathbf{A}$ of $\mathbf{R}$. It is the set of all points of $R$ whose Hausdorff distance to $A$ is less than $\varepsilon$. This set is denoted by $A^{\varepsilon}$.

Jordan curve. It is a continuous one to one application $c$ from $\mathbb{R} / \mathbb{Z}$ into $\mathbb{R}^{2}$. In other terms, a noncrossing curve whose tips are equal.

Jordan curve in $\mathbf{R}$. It is a either a Jordan curve with range in $R$, or a continuous curve $c$ from $] 0,1[$ into $R$ such that or $c(0+)$ and $c(1-)$ exist and are on the boundary of $R$.

Jordan curve theorem. A Jordan curve $c$ divides $\mathbb{R}^{2}$ in two connected components, one bounded which is «enclosed by $c$ s and one unbounded. A Jordan curve in $R$ can be extended in a Jordan curve of $\mathbb{R}^{2}$ in a standard way: one joins $c(0)$ and $c(1)$ by a geodesic of the boundary of $R$. In case where there are two such geodesics, one chooses one of them by some orientation criterion. With these conventions, we still can speak of the area of $R$ «enclosed by $R$ ». 
Isoperimetric inequality in $\mathbb{R}^{2}$ and $\mathbf{R}$. Denote by 0 the area enclosed by a Jordan curve $c$ of $\mathbb{R}^{2}$. Then one has $l(c) \geqslant 2 \pi^{1 / 2}|0|^{1 / 2}$. In the case of a Jordan curve in $R$, the same kind of inequality holds with a smaller constant $C$ :

$$
l(c) \geqslant C|0|^{1 / 2}
$$

Admissible segmentations. $B$ is said admissible if for any $B^{\prime}$ contained in $B$, one has $E\left(B^{\prime}\right) \geqslant E(B)$.

Remark. Any segmentation has an admisible subsegmentation $B^{\prime}$ contained in $B$.

Normal segmentations. In sections 3 and 4 , we shall only consider segmentations made of affine closed curves, called segments. Moreover, we shall suppose, according to the preceding remark, that these segmentations are admissible. All segmentations verifying these conditions will be called normal. Since the segments do not overlap, one sees that such a segmentation gives the length of $B$ as the sum of the lengths of the segments. Finally, a normal segmentation verifies that:

a) $R / S(B)$ has a finite number of connected components. (Indeed, each open segment is in the boundary of at most two connected components).

b) Let $\left(0_{i}\right)_{i \in I}$ be these connected components. Each boundary between $0_{i}$ and $0_{j}$, denoted $b\left(0_{i}, 0_{j}\right)$ is the union of the ranges of a finite set of segments.

c) Each open segment of $B$ is in the boundary of exactly two distinct areas. Indeed, if an open segment has its support in the boundary of only one $0_{i}$, the corresponding curve can be removed from $B$ without changing $u$ and therefore the segmentation is not admissible.

The only reason for which we choose to consider segmentations made of affine curves is that adding a segment to such segmentations cannot create infinitely many crossings.

\section{PASSING TO THE LIMIT IN MINIMIZING SEQUENCES FOR E(B)}

Definition. We shall say that the segmentation $B_{n}$ tends to $B$ if $B_{n}=\left(c_{n}^{i}\right)_{1 \leqslant i \leqslant k}$, $B=\left(c^{i}\right)_{1 \leqslant i \leqslant k}$, and if each $c_{n}^{i}$ tends to each $c^{i}$ uniformly.

Lemma 1. If $B_{n}$ tends to $B$, then $S\left(B_{n}\right)$ tends to $S(B)$ for the Hausdorff distance and $l(B) \leqslant$ liminf $_{n} l\left(B_{n}\right)$.

Proof. Straightforward. Note that it can happen that $l(B)<\Sigma_{k} l\left(c_{k}\right)$. 
Lemma 2. $E(B)$ is a lower semicontinuous functional with respect to the preceding topology.

Proof. Let $B_{n}$ tend to $B$ in the sense defined above. Note that by our conventions, $u_{n}$ and $u$ are automatically defined from $B_{n}$ and $B$. Fix $\varepsilon>0$. For $n$ large enough we have $d\left(B_{n}, B\right) \leqslant \varepsilon$. Denote by $S(B)$ the set of all $x$ in $R$ such that $d(x, S(B)) \leqslant \varepsilon$. Then $S\left(B_{n}\right)$ is contained in $S(B)^{\prime}$ and every connected component $0_{i e}$ of $R / S(B)$ is included in one component $0_{i t t}$ of $R / S\left(B_{n}\right)$. Thus $u_{n}$ is locally constant on every connected component of $R / S(B)^{2}$. Since these connected components are enumerable, we can extract a subsequence of $u_{n}$, still called $u_{n}$, wich converges pointwise in $R / S(B)$. By a diagonal selection argument, one can make $\varepsilon_{k}$ tend to zero and extract another subsequence $u_{n}$ converging pointwise everywhere in $R / S(B)^{r}$. Let $u=\lim u_{n}$. Clearly $u$ is constant in every connected component of $R / S(B)$. Indeed, taken two points $x, y$ of such a component, one has $x$ and $y$ in the same component of $R / S(B)$ for small $\varepsilon$ and therefore $u_{n}(x)=u_{n}(y)$ for large $n$, that is finally $u(x)=u(y)$.

Thus $(u, B)$ is a segmentation of $g$, and moreover by Fatou's lemma one has $\|u-g\| \leqslant \lim \inf \left\|u_{n}-g\right\|$. Then by use of lemma 1 we obtain that $E(B)$ is lower semicontinuous.

\section{ESTIMATES ON THE AREAS AND LENGTHS OF AN ADMISSIBLE SEGMENTATION}

Lemma 3. Let $(u, B)$ be a normal segmentation. Then for any $i$ and $j, l\left(b\left(0_{i}\right.\right.$, $\left.\left.0_{j}\right)\right) \leqslant 4 \min \left(\left|0_{i}\right|,\left|0_{j}\right|\right)$.

Proof. Remove all segments of $b\left(0_{i}, 0_{j}\right)$. We obtain a new segmentation $\boldsymbol{B}^{\prime}$. By the admissibility of $B$, one has: $E\left(B^{\prime}\right) \geqslant E(B)$. Set $u^{\prime}=u$ on any area of $R / S(B)$ different from $0_{i}$ and $0_{j}$ and, assuming for instance that $\left|0_{i}\right|<\left|0_{j}\right|$, define $u^{\prime}$ on the union of $0_{i}$ and $0_{j}$ as the value of $u$ in $0_{j}$. Then clearly we have

$$
E(B) \leqslant E\left(B^{\prime}\right) \leqslant E(B)-l\left(b\left(0_{i}, 0_{j}\right)\right)+4\left|0_{i}\right|
$$

(Indeed, since $|g(x, y)| \leqslant 1$, one has $\left|u^{\prime}-g\right|^{2} \leqslant 4$ on $R$ ).

Lemma 4. Let $B$ be a normal segmentation, and $0_{i}$ the connected component of $R / S(B)$ with minimal measure. Then $0_{i}$ verifies the following inverse isoperimetric inequality:

$$
l\left(b\left(0_{i}\right)\right) \leqslant C^{\prime}\left|0_{i}\right|^{1 / 2} \quad \text { (where } C^{\prime} \text { depends on } R \text { and } g \text { ) }
$$


Proof. Let $J$ be the set of all $j$ such that $b\left(0_{i}\right)$ meets $b\left(0_{j}\right)$. By lemma 3, $l\left(b\left(0_{i}, 0_{j}\right)\right) \leqslant 4\left|0_{i}\right|$, and therefore

$$
\operatorname{Card}(J) \geqslant l\left(b\left(0_{i}\right)\right) / 4\left|0_{i}\right|
$$

Now, each $0_{j}$ verifies $\left|0_{j}\right| \geqslant\left|0_{i}\right|$ and therefore by the isoperimetric inequality in $R$ :

$$
l\left(b\left(0_{j}\right)\right) \geqslant C\left|0_{j}\right|^{1 / 2} \geqslant C\left|0_{i}\right|^{1 / 2}
$$

One clearly has

$$
\Sigma_{j} l\left(b\left(0_{j}\right)\right) \leqslant 2 l(B) .
$$

Thus by (2) $\operatorname{Card}(J) C\left|0_{i}\right|^{1 / 2} \leqslant 2 l(B)$

and by (1) $l\left(b\left(0_{i}\right)\right) C\left|0_{i}\right|^{-1 / 2} / 4 \leqslant 2 l(B)$,

that is: $l\left(b\left(0_{i}\right)\right) \leqslant(8 / C) l(B)\left|0_{i}\right|^{1 / 2}=C^{\prime}\left|0_{i}\right|^{1 / 2}$.

Lemma 5. Let $c$ be a Jordan curve contained in $B$. Then $l(c) \geqslant C\left|0_{i}\right|^{1 / 2}$.

Proof. By the Jordan curve theorem in $R, c$ divides $R$ in two areas. Take a connected component 0 of $R / S(B)$ contained in the area enclosed by $c$. Then one has $l(c) \geqslant C|0|^{1 / 2} \geqslant C\left|0_{i}\right|^{1 / 2}$.

Lemma 6. There exist two real numbers $\varepsilon$ and $\alpha$ such that for any segmentation $B$ with $E(B) \leqslant \min _{B} E(B)+\varepsilon$, then all connected components $0_{i}$ of $R / S(B)$ verify $\left|0_{i}\right| \geqslant \alpha$.

Proof. Define a new segmentation $B^{\prime}$ composed by:

- parametrizations of the four sides of the boundary $b(S)$ of an open square $S$ with center in $0_{i}$ and side $4 C^{\prime}\left|0_{i}\right|^{1 / 2}$.

- For each segment $c$ of $B$, parametrizations of the (at most two) segments whose unión is $S(c) / S$.

By lemma $4,0_{i}$ is contained in the square $S^{\prime}$ with the same center as $S$ and side $2 C^{\prime}\left|O_{i}\right|^{1 / 2}$. Let us now estimate the length of all the curves of $B$ contained in $S$. Let $0_{j}$ be one of the areas which touches $0_{i}$. Thus $b\left(0_{i}\right)$ meets one of the connected components of $b\left(0_{j}\right)$, say $b_{j}$. Since $b_{j}$ is a closed polygon, it contains at least one Jordan curve which is still contained in $S(B)$. Thus by lemma 5 one has $l\left(b_{j}\right) \geqslant C\left|0_{i}\right|^{1 / 2}$. If $b_{j}$ is contained in the square $S$, one still has

$$
l\left(b_{j} \cap S\right) \geqslant C\left|0_{i}\right|^{1 / 2} .
$$

If not, the lenght of the part of the $b_{j}$ contained in $S$ is greater than the distance between $b\left(S^{\prime}\right)$ and $b(S)$, that is:

$$
l\left(b_{j} \cap S\right) \geqslant C^{\prime}\left|0_{i}\right|^{1 / 2} .
$$


Finally, note that a segment $c_{h}$ of $b_{j}$ can be contained in at most one other $b_{k}$ with $k$ in $J$. (Recall that an open segment is in the boundary of exactly two connected components of $R / S(B)$ ). Thus we obtain

$$
2 l(S(B) \cap S) \geqslant \Sigma_{j} l\left(b_{j} \cap S\right) \geqslant \operatorname{Card}(J) \min \left(C, C^{\prime}\right)\left|0_{i}\right|^{1 / 2} .
$$

Using (1) and the isoperimetric inequality for $0_{i}$, we get

$$
\begin{aligned}
& l(S(B) \cap S) \geqslant\left(l\left(b\left(0_{i}\right)\right) / 8\left|0_{i}\right|\right) \min \left(C, C^{\prime}\right)\left|0_{i}\right|^{1 / 2} \\
& l(S(B) \cap S) \geqslant C \min \left(C, C^{\prime}\right) / 8=C^{\prime \prime}
\end{aligned}
$$

Therefore

$$
\begin{aligned}
& l\left(B^{\prime}\right) \leqslant l(B)+l(b(S))-l(S(B) \cap S) \\
& l\left(B^{\prime}\right) \leqslant l(B)+16 C^{\prime}\left|0_{i}\right|^{1 / 2}-C^{\prime \prime}
\end{aligned}
$$

We now proceed to compare the energies of $B$ and the segmentation $B^{\prime}$. Note that $S\left(B^{\prime}\right)$ is obtained from $S(B)$ by adding the boundary of $S$ and removing all parts of curves of $S(B)$ contained in $S$. Associate to $B^{\prime}$ the function $u^{\prime}$ equal to $u$ outside $S$ and equal to, say, 0 on $S$. Then we get.

$$
\left\|u^{\prime}-g\right\|^{2} \leqslant\|u-g\|^{2}+4|S|=\|u-g\|^{2}+64 C^{\prime 2}\left|0_{i}\right|
$$

Using the last two inequalities and the hypothesis

$$
E(B) \leqslant \min _{B} E(B)+\varepsilon \leqslant E\left(B^{\prime}\right)+\varepsilon
$$

we get

$$
E(B) \leqslant E(B)+64 C^{\prime}\left|0_{i}\right|+16 C^{\prime}\left|0_{i}\right|^{1 / 2}-C^{\prime \prime}+\varepsilon
$$

Finally

$$
64 C^{\prime}\left|0_{i}\right|+16 C^{\prime}\left|0_{i}\right|^{1 / 2}-\left(C^{\prime \prime}-\varepsilon\right) \geqslant 0
$$

This ends the proof of lemma 6.

\section{TOPOLOGICAL PROPERTIES OF NORMAL SEGMENTATIONS}

We now wish to prove that if the number of areas is bounded from above, so can be the number of curves of $B$. 
Lemma 7. Given a normal segmentation $B$ defining more than one connected component, there exist two areas $0_{i}$ and $0_{j}$ such that $b\left(0_{i}, 0_{j}\right)$ is a connected curve whose interior is contained in the union of $0_{i}$ and $0_{j}$.

Proof. We use the following well-known facts about the geometry of curves in two dimensions. (The proof of these properties is easy in the case of piecewise affine curves and segmentations.)

a) Let 0 be a bounded open connected set. Then 0 has as external boundary a Jordan curve. It is defined as the boundary of the unbounded component of $\mathbb{R}^{2} /\{0\}$.

b) If a simply connected set is the union of two connected sets $0_{i}$ and $0_{j}$, then $b\left(0_{i}, 0_{j}\right)$ is a connected set.

Now let $c_{0}$ be the Jordan curve of $R$ obtained by taking a parametrization of the boundary of $R$. It is a Jordan curve of $S(B)$ and it encloses all, and therefore at least two, areas of $R / S(B)$. If $R$ is the union of exactly two areas $0_{i}$ and $0_{j}$, then by property $\left.b\right) b\left(0_{i}, 0_{j}\right)$ is connected and since $B$ is admissible it is clearly a piecewise affine one to one curve. If not, choose two areas enclosed by $c_{0}$ and having a common boundary of positive length. Let $c_{1}$ be the external boundary of the union of these areas. We iterate the process and obtain a sequence of Jordan curves in $R, c_{0}, c_{1}, c_{2}, \ldots$ where each curve is enclosed by the preceeding. Since the number of areas is finite, one sees easily that this process must finish at some $c_{k}$ enclosing exactly two areas, and then we use again $b$ ) and the admissibility of $B$.

Lemma 8. Let $B$ a normal segmentation. Then there exists another segmentation $B^{\prime}$ with $S(B)=S\left(B^{\prime}\right)$ and such that the number of curves of $B^{\prime}$ is strictly less than the number of areas of $R / S\left(B^{\prime}\right)$.

Proof. Let $c_{1}$ be a curve as given by lemma 7. Define $B_{1}$ as $B$ minus the segments of $c_{1}$. Then $B_{1}$ is still a normal segmentation and has one connected component less than $B$. Moreover, $B_{1}$ is admissible. Indeed, the boundary of $0_{i}$ and $0_{j}$ has been completely removed by removing $c_{1}$. Iterating this process we define a sequence of curves $c_{1}, c_{2}, \ldots, c_{h}, \ldots$ until some $B_{k}$ defines only one connected component, $R$. Since $B_{k}$ is admissible, that means that $S\left(B_{k}\right)$ is empty. Thus $B^{\prime}=\left(c_{h}\right)_{h \leqslant k}$ is a segmentation which has the required properties.

Lemma 9. Let $\alpha$ be the number of areas of a normal $B$. Then the number $\gamma$ of geometrical crossings and the number $\beta$ of geometrical curves of $B$ verify:

$$
\gamma \leqslant 2(\alpha-1), \quad \text { and } \quad \beta \leqslant 3(\alpha-1)-2
$$

Proof. The curves $c_{k}$ of the preceding proof contain at most two geometrical crossings of the segmentation $B_{k-1}$, at their ends. Thus passing 
from $B_{k-1}$ to $B_{k}$ eliminates at most two geometrical crossings. By iteration we obtain the first announced inequality. Let now $\beta_{k}$ be the number of geometrical curves of $B_{k}$. Note that the support of $c_{k}$ is one of them. One has clearly

$$
\beta_{k} \leqslant \beta_{k-1}-3
$$

Indeed, if no crossing point disappears by removing $c_{k}$, the only geometrical curve which is suppressed is $c_{k}$. If a crossing point contained in $R$ disappears, this means that exactly three geometrical curves meet at this point, one of which is $c_{k}$. By removing $c_{k}$ we transform both other geometrical curves in one. If a crossing point in the boundary of $R$ disappears, that means that $c_{k}$ is the only geometrical curve with tip at this point and no other geometrical curve disappears. Thus removing $c_{k}$ from $S\left(B_{k}\right)$ suppresses at most three geometrical curves, one for each tip and $c_{k}$ itself. Take into account that removing the last $c_{k}$, which is Jordan in $R$, suppresses only one geometrical curve. This yields the announced estimate.

\section{EXISTENCE AND REGULARITY PROPERTIES OF OPTIMAL SEGMENTATIONS}

We beging by showing that all properties proved above for normal segmentations remain valid as we pass to the limit. From the previous paragraphs, it is clear that we can choose a sequence $B^{n}$ of normal segmentations with the following properties:

a) $B^{n}=\left(c_{k}^{n}\right)_{k}$ is made of a constant number of one to one piecewise affine curves from ]0,1[ into $R$ whose tips lay all in a finite set of points, $A=\left(a_{i}^{n}\right)_{i}$.

b) The points $a_{i}^{n}$ are the geometrical crossings of $B^{n}$.

c) Each sequence $a_{i}^{n}$ converges to a point $a_{i}$ of the closure of $R$.

d) Each curve $c_{k}^{n}$ converges uniformly to a Lipschitz curve $c_{k}$ in the closure of $R$.

e) The sequence $E\left(B^{n}\right)$ tends to $\operatorname{Min}_{B} E\left(B^{n}\right)$, this minimum being taken among all $C^{1}$ segmentations.

Properties $a$ ) and $b$ ) are obtained by reparametrizing each $B^{n}$ so that each curve is made of a chain of segments where only the first and the last contain a geometrical crossing at the tip. In other terms, we reparametrize $B^{n}$ so that all curves of $B^{n}$ have geometrical curves as supports. By Lemma 9 we get an uniformly bounded number of curves and crossing points which we can make constant by extracting a subsequence. 
Properties $c$ ) and $d$ ) are obtained by Acoli-Arzela theorem and extraction of a subsequence.

Property $e$ ) is due to the straightforward fact that a $C^{1}$ segmentation can be replaced by a piecewise affine one whose energy is arbitrarily close.

Set $B=\left(c_{k}\right)_{k}$. Since parts of the curves can a priori be contained in the boundary of $R, B$ cannot be called a segmentation. We shall now prove that it is a smooth segmentation.

Lemma 10. The only geometrical crossings of $B$ are the $a_{i}$.

Proof. Assume that a new geometrical crossing $a$ appears as we pass to the limit. We first consider the case where $a$ is in $R$. Fix $\varepsilon$ very small so that $\varepsilon^{2} \ll \mathrm{C} \varepsilon$ for all the constants $C$ which we shall introduce in the following. In particular, we shall neglect the terms of order $\varepsilon^{2}$ in all the following estimates. (They will be neglected as they correspond to area integrals in disks of radius $\varepsilon$ if we "gain" some length term of order $C \varepsilon$ by changing the segmentation in this disk). Assume moreover that $\varepsilon<\min _{i}\left\|a-a_{i}\right\|$ and take $n$ large enough such that

$$
E\left(B^{n}\right) \leqslant \operatorname{limin} f_{n} E\left(B^{n}\right)+\varepsilon^{2}, \quad\left\|a_{i}^{n}-a_{i}\right\| \leqslant \varepsilon^{2}, \quad\left\|c_{k}^{n}-c_{k}\right\| \leqslant \varepsilon^{2}
$$

Since the curves $c_{k}^{n}$ are piecewise affine, the number of their crossings with the boundary of $D$ is finite. Moreover, one can associate by pairs these crossings on each curve: to the first crossing of $c_{k}^{n}$ with $b(D)$, we associate the second along the parametrization of $c_{k}^{n}$, to the third the fourth and so on. These pairs of points are clearly the boundaries of all maximal pieces of curves of $B$ with supports contained in $D$.

Let us now replace each one of these pieces of curves by an affine curve. Since the $c_{k}^{n}$ do not cross in $D$, these affine curves do not cross. By this smoothing the connected components of $R / S(B)$ remain unchanged outside $D$ and the length of the curves goes down. Call $B^{\prime}$ the segmentation obtained after adding to $B$ the affine curves in $D$ defined above, removing from $B$ the curves meeting $D$, and adding the reparametrizations of the parts outside $D$ of these curves.

We still have $E\left(B^{\prime}\right) \leqslant \min _{B} E(B)+C \varepsilon^{2}$. (In fact we can take $C=4 \pi+1$ ). Now consider two affine curves $[u, v]$ and $[x, y]$ of $B^{\prime}$ which have been obtained by smoothing two parts of curves of $B^{n}$ passing at a distance less than $\varepsilon^{2}$ from $a$. Since $a$ is a geometrical crossing of $B$, such curves exist for large $n$. The lengths of the corresponding pieces of curves are greater than $2 \varepsilon-2 \varepsilon^{2}$ since they pass at a distance less than $\varepsilon^{2}$ from the center $a$ of $D$. Moreover, the smoothing cannot make these lengths decrease more than $\varepsilon^{2}$. 
Thus $[u, v]$ and $[x, y]$ are in fact nearby diameters of $D$ and they do not cross. By eventually echanging $u$ and $v$ we obtain that $\|u-x\|$ and $\|v-y\|$ are less than $C \varepsilon^{2}$. The contradiction comes by modifying $B^{\prime}$ as follows: we add to $B^{\prime}$ the segments $[u, x]$ and $[v, y]$ and remove one of the segments $[u, v]$ or $[x, y]$. This does not modify the connected components outside $D$. Thus we have substracted from $E\left(B^{\prime}\right)$ a length of order $2 \varepsilon$, which is a contradiction.

If now a new geometrical crossing appears in $B$ on the boundary of $R$ : we leave the proof to the reader.

Lemma 11. The geometrical curves of $B$ are $C^{1}$.

Proof. Let $c$ be a curve of $B$, parametrized by the length $s$ along the curve. If we replace the curve between two points $c(s)$ and $c(s+\varepsilon)$ by the corresponding segment, the isoperimetric inequality proves that the areas of both connected components touching $c$ changes less than $C \varepsilon^{2}$. B being minimal, we obtain: $\|c(s+\varepsilon)-c(s)\| \geqslant \varepsilon+C \varepsilon^{2}$, and $C$ depends only on $g$ and $R$. One deduces easily from this relation that $c$ is $C^{1}$ and that $c^{\prime}$ is Lipschitz with constant less than $2 C$.

Lemma 12. The geometrical crossing points of $B$ are as announced in the main theorem of the introduction.

Proof. Assume by contradiction that more than three curves arrive to a geometric crossing point $a$ or that exactly three arrive, but with angle different from $120^{\circ}$. Then two of these curves form an angle strictly less than $120^{\circ}$. Let $\varepsilon$ very small and $D$ as above. Call $u$ and $v$ the first crossings of both curves with the boundary of $B$ and $w$ the unique point such that the lines $w u$, $w v$, wa have $120^{\circ}$ angles. Remove from the segmentation the pieces of curves $a u$ and $a v$ and add the segments $w u, w v, w a$. This modification does not alter the connected components outside $D$, changes the area terms with order $\varepsilon^{2}$ and reduces the length of $B$ with order $\varepsilon$ : impossible.

Conclusion. By Lemma 1, we have $E(B) \leqslant \operatorname{limin} f_{n} E\left(B^{n}\right)$ and since $B$ is a $C^{1}$ segmentation, it is clear that $E$ achieves its minimum at $B$.

Lemma 13. If $g$ is continuous, each interior point $x$ of a curve $c$ verifies the following Euler-Lagrange equation:

$$
\text { (Curvature of } c \text { at } x)=\left(\text { jump of }(u-g)^{2} \text { accross } c \text { at } x\right. \text { ) }
$$

Proof. Comes from a classical perturbation method in elasticity theory. See Blat and Morel [1]. 
Aknowledgement. This work has been completed while the first author was visiting the SISSA (Trieste), the University of Baleares and the Complutense University of Madrid. He thanks José Blat, Ennio De Giorgi and Pierre Baras for valuable conversations.

\section{References}

[1] BLAT, J. and MOREL, J. M. Elliptic problems in image segmentation and their relation to fracture theory. To appear in Proceedings of the Int. Conf. on nonlinear elliptic and parabolic problems, Nancy 1988.

[2] CONGEDO, G. and TAMANINI, I. Note sulla regolarita dei minimi di funzionali del tipo dell'area (preprint, Universita di Trento, Italy).

[3] De Giorgi, E. and Ambrosio, L. Un nuovo tipo di funzionale del calcolo delle variazioni. To appear in Atti Acad. Naz. Lincei Rend. Cl. Sci. Fis. Mat. Natur.

[4] GEMAN S. and GEMAN D. Stochastic relaxation, Gibbs distributions and the Bayesian restoration of images, IEEE PAMI 6 (1984).

[5] MODICA, L. Gradient theory of phase transitions with boundary contact energy. Ann Inst. Poincaré, vol. 4, n. ${ }^{\circ} 5,1987$, pp. 487-512.

[6] Mumford, D. and Shah, J. Preprint, Harvard University, Cambridge, Massachussets, USA.

[7] MUMFORD, D. and SHAH, J. Boundary detection by minimizing functionals, IEEE Conference on Computer Vision and Pattern Recognition, San Francisco, 1985.

[8] RosenfelD, A. and KAK, A. C. Digital picture processing. Computer Science and Applied Mathematics, Academic Press, 1982.

[9] Yang Wang. Preprint, Aiken Lab., Harvard University.

Ceremade, Univ. Paris-Dauphine

Place du Maréchal de Lattre

75775 Paris Cedex 16

France

\author{
SISSA \\ Viale Miramare 325 \\ 35014 Trieste \\ Italy
}

Recibido: 6 de septiembre de 1988 